\title{
Hyper-Spectral Networking Concept of Operations and Future Air Traffic Management Simulations
}

\author{
Paul Davis \\ Architecture Technology Corporation \\ Campbell, CA, USA \\ pdavis@atcorp.com
}

\author{
Benjamin Boisvert \\ Architecture Technology Corporation \\ Hampton, VA, USA \\ bboisvert@atcorp.com
}

\begin{abstract}
The NASA sponsored Hyper-Spectral Communications and Networking for Air Traffic Management (ATM) (HSCNA) project is conducting research to improve the operational efficiency of the future National Airspace System (NAS) through diverse and secure multi-band, multi-mode, and millimeter-wave (mmWave) wireless links. Worldwide growth of air transportation and the coming of unmanned aircraft systems (UAS) will increase air traffic density and complexity. Safe coordination of aircraft will require more capable technologies for communications, navigation, and surveillance (CNS). The HSCNA project will provide a foundation for technology and operational concepts to accommodate a significantly greater number of networked aircraft. This paper describes two of the HSCNA project's technical challenges. The first technical challenge is to develop a multi-band networking concept of operations (ConOps) for use in multiple phases of flight and all communication link types. This ConOps will integrate the advanced technologies explored by the HSCNA project and future operational concepts into a harmonized vision of future NAS communications and networking. The second technical challenge discussed is to conduct simulations of future ATM operations using multi-band/multi-mode networking and technologies. Large-scale simulations will assess the impact, compared to today's system, of the new and integrated networks and technologies under future air traffic demand.
\end{abstract}

Keywords-aviation networking concept of operation, communication link modeling, air traffic management simulation, autonomous aircraft operations, unmanned aircraft systems

\section{INTRODUCTION}

The volume of passenger and cargo air traffic will continue to increase over time. As unmanned aircraft systems (UAS) become integrated in the National Airspace System (NAS) their use is predicted to grow significantly [1]. The resulting increase in air traffic density and complexity has the potential to adversely impact the safety and efficiency of the NAS. Safe and efficient operations, especially as aircraft become increasingly more autonomous, will depend on highly-reliable and efficient communications and networking between aircraft, air navigation service providers, and aircraft operators. Airborne communication networks will need to handle data rates that are tens to thousands of times larger than in use today. Research is needed in multiple areas to realize the networking capabilities necessary to support future air traffic demand.
A three year Hyper-Spectral Communications and Networking for Air Traffic Management (ATM) (HSCNA) research project, described in [2], intends to dramatically enhance the capabilities of aviation communication and networking systems. Research will focus on aeronautical communications, networking, and ATM, including aspects of navigation and surveillance, for both manned and unmanned aircraft. Six technical challenges (TC), derived from the project's high-level strategic and research outcomes, have been defined. They are:

TC1: Develop a multi-band networking concept of operations (ConOps) for use in multiple phases of flight and all communication link types, e.g., air-ground, air-air. Identify opportunities for improved performance and specific physical layer/data link layer/networking techniques required, taking into consideration future vehicle types, missions, frequency bands, and traffic forecasts.

TC2: Quantify the capacity and coverage of existing aviation (and adjacent) frequency bands and technologies, quantify their shortcomings and mid- to far-term ( 2035) improvements, and assess growth potential. Example techniques to be investigated include application of spectrally, temporally, and spatially efficient links, non-traditional aviation resources (modes) such as existing commercial radio towers, and advanced processing such as interference cancellation. Assess the performance potential of truly revolutionary technologies e.g., free-space optical wireless between aircraft, a vast aviation database aboard every aircraft, free and abundant energy sources for all aircraft, accurate and ubiquitous short-term numerical weather prediction, etc., in terms of gains in ATM capacity, flexibility, and safety.

TC3: Build analysis and simulation software toolboxes and prototypes to enable assessment of adaptive link and network performance (physical layer, data link layer, and network reliability, latency, etc.) over multiple frequency bands using multiple communication modes in a hyper-spectral network. Develop a small UAS testbed with multiple networked UAS to validate multi-band and multi-mode communication performance. Adapt physical layer/data link layer/networking routines for integration into ATM simulations.

TC4: Quantify capacity and efficiency gains of widespread millimeter-wave (mmWave) wireless airport subnetworks. Measure and model example channels and validate prototype

This work is supported by the National Aeronautics and Space Administration under Federal Award ID number NNX17AJ94A. 
mmWave system(s) in example airport network operations. Extend mmWave application to small UAS.

TC5: Develop novel jammer and unauthorized UAS detection/localization techniques to detect and track any unauthorized UAS or jammer that enters any restricted zone.

TC6: Develop a realistic and comprehensive ATM simulation capability to assess the gains of multi-band/multimode and mmWave networking in terms of data link performance per aircraft, supportable traffic density, multivehicle collaboration, and operational benefits. Cover a range of timescales from current to long-term and a range of operational phases from surface operations to enroute.

TC2 and TC3 are discussed in more detail in [2], TC4 in [3], and TC5 in [4]. The focus of this paper is on the research approach for addressing TC1 and TC6.

\section{CONCEPT OF OPERATIONS}

Safe and efficient management of future air traffic requires improved communications, navigation, and surveillance (CNS) technologies. Sophisticated sensors onboard the aircraft and outside the aircraft, will be used for performing rapid and varied situational and environmental awareness measurements, allowing a high level of aircraft autonomy. However, movement of information among NAS entities will still be required for coordination and collaboration, especially in airspaces with higher densities of operations. Increasingly autonomous aircraft will generate and consume more and more information, further increasing the demand on networking. Because all CNS requires wireless electromagnetic signaling among the participating entities, multiple effective, efficient, and highly-reliable communications technologies will be required to service the diverse range of aircraft sizes and capabilities.

Technical Challenge 1 is Develop a Multi-band Networking ConOps for use in Multiple Phases of Flight and all Communication Link Types. Future hyper-spectral and multimode networking has the potential to enable and profoundly benefit NAS operations. Entirely new modes of operation, such as fully autonomous strategic trajectory planning, negotiation and execution, can be supported.

The purpose of the HSCNA ConOps is to provide the vision and operational context for the research. The ConOps describes how the explored technologies are applied, the feasibility, and the anticipated benefits. The ConOps describes the baseline NAS networking capabilities, the future concepts and technologies such as multi-band, multi-mode network technologies, mmWave systems, jammer detection, and CNS capabilities. Operational scenarios describe and justify, including estimates and assumptions, how these future concepts are applied. For example a future concept may be a transformational network technology which is applied to a network-connected UAS operations scenario. The ConOps also includes the basis and approach for forecasting far-term air traffic demand: number of flights, types of vehicles, and missions.
The ConOps lays the foundation for and is impacted by several of the other technical challenges. TC3 Multi-band Multi-Mode Link Design develops the link physical layer/data link layer/network designs, which the ConOps summarizes and sets in an operational context. TC6 Conduct Simulations to Assess Future ATM Operations using Multi-band/Multi-mode Networking and Technologies simulates network performance under future air traffic conditions, including operational scenarios developed for the ConOps; and incorporates the techniques of TC3 and TC4 mmWave Channel Propagation Modeling and Air Interface and Medium Access Control Design for Airport Operations and Small UAS.

Developed during the first year of the project, the ConOps will encapsulate the research team's aggregate, harmonized vision of future NAS communications and networking. The concepts and operational scenarios are developed through multiple rounds of collaborative input, review, and refinement. Related work, such as an ongoing NASA sponsored, Boeingled study researching Integrated UAS CNS Architecture [5] [6], will be reviewed and incorporated as appropriate. The ConOps is peer reviewed and will undergo a final review by NASA. A successful ConOps will be comprehensive yet tractable and is both traceable and flexible enough to allow future growth and novel/disruptive technical solutions.

\section{SIMULATION ASSESSMENT}

TC6 is to Conduct Simulations to Assess Future ATM Operations using Multi-band/Multi-mode Networking and Technologies. Simulation under anticipated operational conditions is an important part of assessing the networking technologies and directing them toward beneficial system improvements. Air traffic simulations can illustrate, at a high but realistic level, future aircraft density, movement, and associated network requirements. The HSCNA simulations will provide an operational context for the networking technologies. For example using mmWave capabilities on the airport surface versus for air-to-air networking. Simulations contrast what can be achieved with, and the performance of, future networking technologies against the current NAS. Performance is especially important when considering a very dense network and/or one that uses a very large frequency band, as in the hyper-spectral case.

To assess the operational impacts of the ConOps requires constructing models and a choosing a simulation platform. The challenge is selecting and configuring a comprehensive yet efficient software platform and set of modules to represent the physical layer/data link layer/networking techniques and provide an appropriate level of accuracy. Several air traffic simulators, including NASA's Airspace Concepts Evaluation System (ACES) [7] and Airspace and Traffic Operations Simulation (ATOS) [8], can model current and near-term CNS systems. However, modeling future networking concepts will require significant development within each of these simulation environments. 


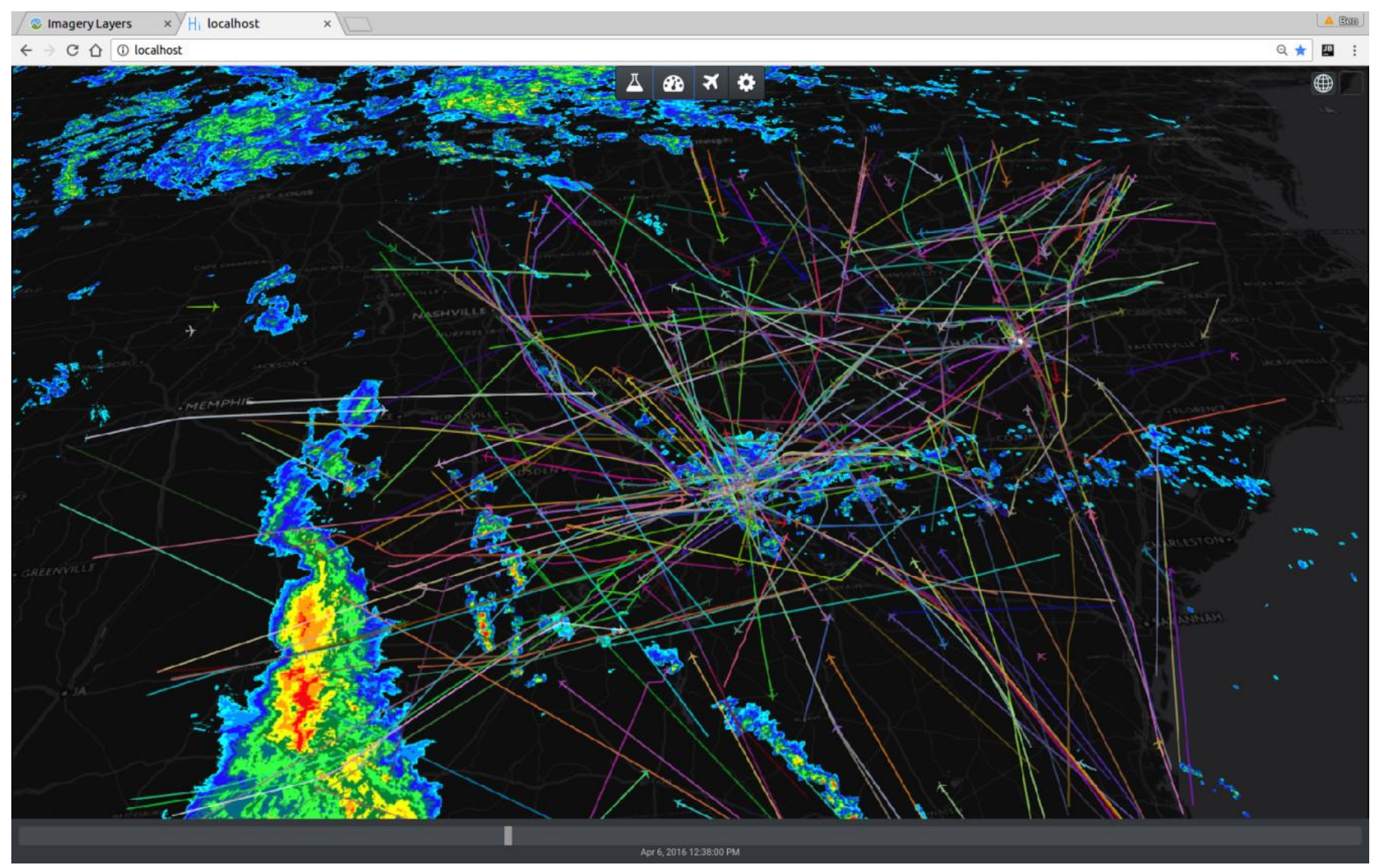

Fig. 1. PNP provides a fast-time simulation environment for assessing the ability of new communication links to support future ATM operations. Regional or NAS-wide simulations of current day and future traffic scenarios support evaluation under a range of air traffic and weather conditions.

Architecture Technology Corporation's Probabilistic NAS Platform (PNP) [9] provides an appropriate level of fidelity and features, is easy to extend, easy to operate, and will be used for conducting rapid assessments of the HSCNA ConOps. PNP, shown in Fig. 1, models enroute, terminal, and airport surface operations and is capable of performing both regional and NAS-wide simulations. Existing PNP plugins provide the ability to model separation assurance, arrival/departure metering, runway configuration management, wake vortex separation, tactical and strategic rerouting due to congestion and weather.

For HSCNA, PNP will be extended to incorporate elements of the ConOps and TCs 2, 3, 4 and potentially TC5. PNP will model communication utilization based on the vehicle type (e.g., level of equipage, or automation) and the phase of flight (e.g., enroute, terminal, surface). This will produce network loading and performance information (e.g., link error rates, latency, etc.) over time and by operational region (e.g., terminal airspace). Model accuracy will be compared to higher fidelity physical layer/data link layer/networking results generated by the other Technical Challenges.

An approach for assessing communication performance under different traffic conditions is to explicitly simulate each message sent between each aircraft and ground station. The content of the messages are updated (just as they would be in the real world) based on the phase of flight, aircraft location, type of aircraft, equipage, networking concept, communications link, and operational conditions. Although this approach allows for a fairly realistic representation of a future communications network, it adds a significant computational load to the assessment platform, limiting the number of simulations that can be conducted. Additionally this higher level of modeling fidelity is not necessary for the types of assessments that HSCNA needs to perform.

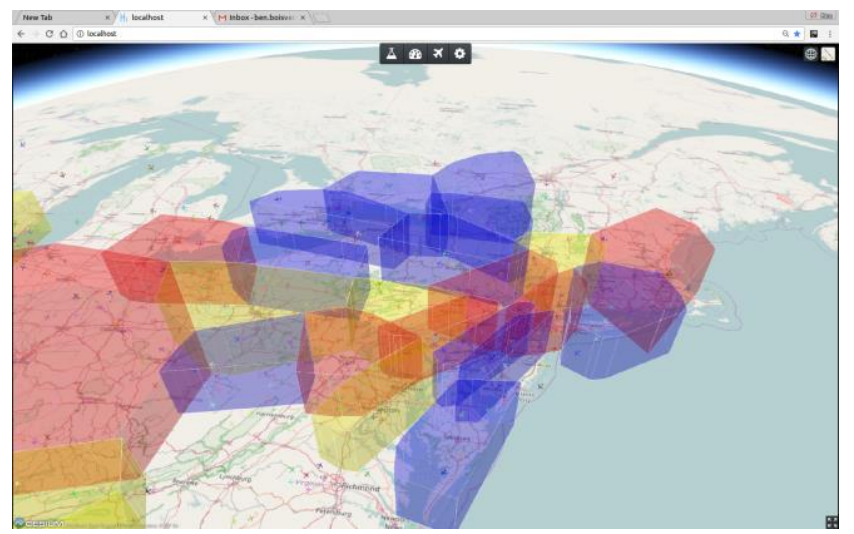

Fig. 2. The PNP approach for computing airspace density is leveraged to compute performance of future aviation networks. 
A better suited approach for HSCNA concept assessment is to use PNP to generate the traffic and operational conditions at discrete points in time and use that information to compute the communication network metrics throughout the simulation. In other words, the core PNP simulator determines the locations of and mixture of aircraft (including UAS) for each airspace partition (e.g., enroute sectors), for specific time bins (e.g., every 5 minutes), and specific operational conditions (e.g., convective weather). PNP's airspace sector loading capability, illustrated in Fig 2, computes airspace densities and can be leveraged. A new PNP multi-mode/multi-band communication network plugin will be developed. This plugin will include models for current ATM communication links and also for the future communication links. The plugin will integrate with PNP to obtain the current simulation state and compute the network performance metrics. The network performance over time can be summarized as well as partitioned by region (including airport surface), vehicle type, communication type, etc. The network performance will be evaluated to determine how well it can support the specific operational scenarios.

Architecture Technology's AvDemand ${ }^{\mathrm{TM}}$ [10], has generated input for many NAS-wide and regional simulations of future ATM concepts including UAS operations [11], and will be used to generate and validate future flight demand sets

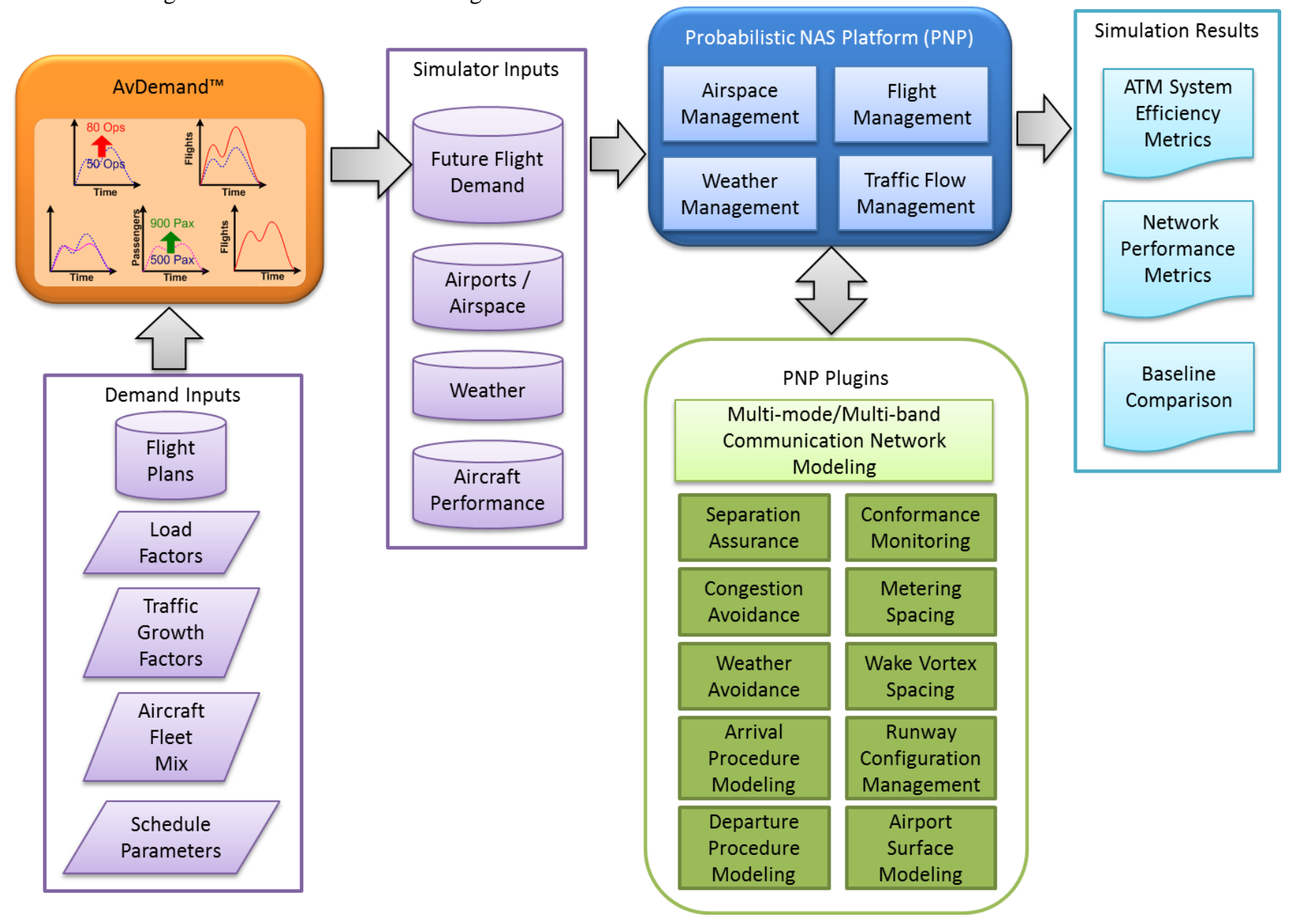

(demand includes: schedules, routes, vehicle types, equipage, etc.) AvDemand ${ }^{\mathrm{TM}}$ will generate both current and future demand sets for the simulations. A baseline assessment of today's air traffic operations with current communication applications (e.g., Data Comm, ACARS, ADS-B) and current links will be conducted using PNP. The same traffic scenario will then be assessed using the new networking links to determine relative performance. This comparative analysis will be extended to additional scenarios representing different assumptions about the future air traffic (e.g., significant increase in passengers, autonomous UAS operations, etc.). Additional operational capabilities and conditions, such as weather, will also be incorporated into the simulations. For example, network-based trajectory negotiation may enable more direct routes than is currently typical. Weather may impact traffic densities and alter communication throughput. The initial focus will be on regional scenarios with representative operations. Realistic and accurate simulation of localized operations should produce more relevant results than a less realistic simulation of NAS-wide operations. NAS-wide assessments, using AvDemand ${ }^{\mathrm{TM}}$ and PNP will be conducted as needed.

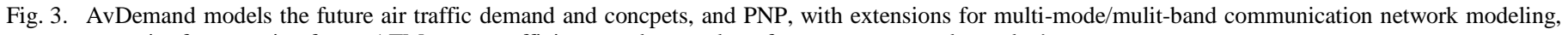
computes metrics for assessing future ATM systtem efficiency and network performance compared to today's system. 
The HSCNA simulation environment is depicted in Fig 3. Historical flight plans will be used for generating the baseline and future flight demand sets. Flight plans for UAS, which are not prevalent in current day flight data, will be introduced by 1) replacing a subset of conventional aircraft flight plans with UAS aircraft, and 2) adding new flight plans for nominal UAS missions that are not present in today's NAS. Through AvDemand $^{\mathrm{TM}}$ the mixture of UAS flights that are replacements/additions to currently flown missions, and those that are entirely new types of missions can be controlled. A flight demand set, along with weather and other simulator inputs comprise a scenario for a specific simulation. PNP can be configured as needed, using any of the available plugins, to model specific functions that impact the ATM system. A new Multi-mode/Multi-band Communication Network plugin will utilize services provided by the core PNP simulator as well as by those provided by other plugins. Standard ATM efficiency metrics, such as aircraft throughput and delay will be generated. The Multi-mode/Multi-band Communication Network plugin will generate the metrics to evaluate the performance of the various links. The metrics for each future simulation can be compared to the corresponding baseline metrics to provide a relative benefit assessment of the new communication link under future air traffic and ATM operational scenarios.

The HSCNA simulation environment is intended to provide the research community with a platform to continue advancements in this discipline beyond the scope of this project.

\section{SUMMARY}

The HSCNA project is conducting foundational research into new communications technologies for future aviation networks. As part of this research a ConOps will be developed that integrates and provides the context for applying these advanced networking technologies to the operational challenges caused by increasing levels of automation and air traffic. The ConOps guides simulation-based assessments that will evaluate the impact and benefits of integrated multiband/multi-mode networking on the safety and efficiency of future NAS operations.

\section{ACKNOWLEDGMENT}

The authors wish to thank the project's Principal Investigator, Dr. David Matolak, University of South Carolina.
We would also like to acknowledge our co-investigators, Dr. Ismail Guvenc, North Carolina State University and Dr. Hani Mehrpouyan, Boise State University.

\section{REFERENCES}

[1] US Department. of Transportation, "Unmanned Aircraft System (UAS) Service Demand 2015-2035: Literature Review and Projections of Future Usage," Technical Report, v.1.0, DOT-VNTSC-DoD-13-01, February 2014.. London, vol. A247, pp. 529-551, April 1955.

[2] D. Matolak, "Hyper-spectral communications, networking \& ATM as foundation for safe and efficient future flight: transcending aviation operational limitations with diverse and secure multi-band, multi-mode, and mmWave wireless links: project overview, aviation communications and new signaling," 2017 Avionics Systems Conference (DASC), St. Petersburg, FL, 17-21 September 2017.

[3] H. Mehrpouyan, "Millimeter wave systems for airports and short-range aviation communications," 2017 Avionics Systems Conference (DASC), St. Petersburg, FL, 17-21 September 2017.

[4] I. Guvenc, "Detection, localization, and tracking of unauthorized UAS and jammers," 2017 Avionics Systems Conference (DASC), St. Petersburg, FL, 17-21 September 2017.

[5] F. Templin, R. Jain, G. Sheffield, P., Taboso-Ballesteros, and D. Ponchak, "Considerations for an integrated UAS CNS architecture," 2017 Integrated Communications Navigation and Surveillance (ICNS) Conference, Herndon, VA, April 2017.

[6] F. Templin, R. Jain, G. Sheffield, P., Taboso-Ballesteros, and D. Ponchak, "Requirements for an integrated UAS CNS architecture," 2017 Integrated Communications Navigation and Surveillance (ICNS) Conference, Herndon, VA, April 2017.

[7] G. Couluris, G., Hunter, M. Blake, K., Roth, D. Sweet, A. Stassart, J. Phillips, and A. Huang, "National Airspace System simulation capturing interactions of air traffic management and flight trajectories," AIAA Modeling and Simulation Technologies Conference, AIAA-2003-5597, Austin, TX, August 2003.

[8] D. Finkelsztein, T. Lung, R. Vivona, J. Bunnell, D. Mielke, and W. Chung, "Airspace and traffic operations simulation for distributed ATM research and development," AIAA Modeling and Simulation Technologies Conference and Exhibit, AIAA 2005-6488, San Francisco, CA, August 2005.

[9] K. Ramamoorthy, B. Boisvert, and G. Hunter, "A real-time probabilistic TFM evaluation tool," AIAA Digital Avionics Systems Conference (DASC), Portland, OR, 2006.

[10] A. Huang and D. Schleicher, "A flexible demand generation application for NextGen concept evaluation," AIAA Modeling and Simulation Technologies Conference and Exhibit, AIAA-2007-6876, Hilton Head Island, SC, 2007.

[11] A. Saxena, I. Roychoudhury, C. Neukom, G. Pisanich, A. Huang, and D. Tran, "Evaluating the impact of deploying unrestricted unmanned aircraft systems in the national airspace," 2012 AIAA Infotech@ Aerospace, Garden Grove, CA, June 2012. 